\title{
Is the water-Pt(111) interface ordered at room temperature?
}

\author{
Mikkelsen, August E. G.; Schiøtz, Jakob; Vegge, Tejs; Jacobsen, Karsten W.
}

Published in:

Journal of Chemical Physics

Link to article, DOI:

$10.1063 / 5.0077580$

Publication date:

2021

Document Version

Peer reviewed version

Link back to DTU Orbit

Citation $(A P A)$ :

Mikkelsen, A. E. G., Schiøtz, J., Vegge, T., \& Jacobsen, K. W. (2021). Is the water-Pt(111) interface ordered at room temperature? Journal of Chemical Physics, 155(22). https://doi.org/10.1063/5.0077580

\section{General rights}

Copyright and moral rights for the publications made accessible in the public portal are retained by the authors and/or other copyright owners and it is a condition of accessing publications that users recognise and abide by the legal requirements associated with these rights.

- Users may download and print one copy of any publication from the public portal for the purpose of private study or research.

- You may not further distribute the material or use it for any profit-making activity or commercial gain

- You may freely distribute the URL identifying the publication in the public portal

If you believe that this document breaches copyright please contact us providing details, and we will remove access to the work immediately and investigate your claim 


\section{Is the water-Pt(111) interface ordered at room temperature?}

Accepted Manuscript: This article has been accepted for publication and undergone full peer review but has not been through the copyediting, typesetting, pagination, and proofreading process, which may lead to differences between this version and the Version of Record.

Cite as: J. Chem. Phys. (in press) (2021); https://doi.org/10.1063/5.0077580

Submitted: 04 November 2021 • Accepted: 09 November 2021 • Accepted Manuscript Online: 15 November 2021

August Edwards Guldberg Mikkelsen, (iD) Jakob Schiotz, (i) Tejs Vegge, et al.
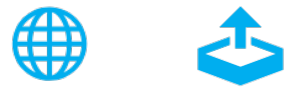

\section{ARTICLES YOU MAY BE INTERESTED IN}

When do short-range atomistic machine-learning models fall short?

The Journal of Chemical Physics 154, 034111 (2021); https://doi.org/10.1063/5.0031215

Jacob's ladder of density functional approximations for the exchange-correlation energy AIP Conference Proceedings 577, 1 (2001); https://doi.org/10.1063/1.1390175

Machine learning for interatomic potential models

The Journal of Chemical Physics 152, 050902 (2020); https://doi.org/10.1063/1.5126336

Challenge us.

What are your needs for periodic signal detection?
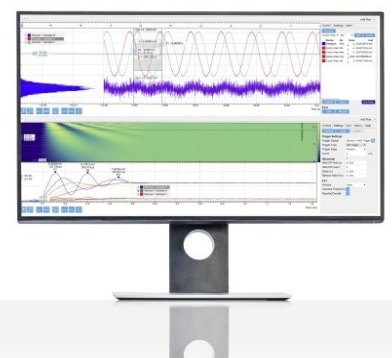

- Zurich - Instruments

J. Chem. Phys. (in press) (2021); https://doi.org/10.1063/5.0077580

(c) 2021 Author(s). 


\title{
Is the water/Pt(111) interface ordered at room temperature?
}

\author{
August E. G. Mikkelsen, ${ }^{1}$ Jakob Schiøtz, ${ }^{2}$ Tejs Vegge, ${ }^{1,}$ a) and Karsten W. Jacobsen ${ }^{2}$ \\ ${ }^{1)}$ Department of Energy Conversion and Storage, Department of Physics, Technical University of Denmark, DK-2800 Kgs. Lyngby, \\ Denmark \\ ${ }^{2)}$ CAMD, Department of Physics, Technical University of Denmark, DK-2800 Kgs. Lyngby, \\ Denmark
}

(*Electronic mail: teve@dtu.dk)

(Dated: 12 November 2021)

The structure of the water/Pt(111) interface has been a subject of debate over the past decades. Here, we report the results of a room temperature molecular dynamics study based on neural network potentials, which allow us to access long time scale simulations while retaining ab initio accuracy. We find that the water/Pt(111) interface is characterized by a double layer composed of a primary, strongly bound adsorption layer with a coverage of $\sim 0.15 \mathrm{ML}$, which is coupled to a secondary, weakly bound adsorption layer with a coverage of $\sim 0.58 \mathrm{ML}$. By studying the order of the primary adsorption layer we find that there is an effective repulsion between the adsorbed water molecules, which gives rise to a dynamically-changing, semi-ordered interfacial structure, where the water molecules in the primary adsorption layer are distributed homogeneously across the interface forming frequent hydrogen bonds to water molecules in the secondary adsorption layer. We furthermore show that these conclusions are beyond the time scales accessible to ab initio molecular dynamics.

\section{INTRODUCTION}

Water-metal interfaces are ubiquitous in nature and relevant to a broad range of technological areas such as corrosion, electro-catalysis, environmental chemistry and lubrication 233 . Over the past decades, this has led to a huge interest within the scientific community on developing accurate theoretical and computational models that can help to understand the relevant physical and chemical processes that take place in these systems.

Within the field of electro-catalysis, water-metal interfaces are particularly important due to the widespread use of aqueous electrolytes. In this context, the water/Pt interface is often considered a "benchmark" system, as Pt-based electrodes are used extensively in modern electrochemical devices such as PEM fuel cells ${ }^{4}$. Because of this a detailed theoretical understanding of the water/Pt interface has been of particularly high interest, and this has resulted in a plethora of computational studies at different levels of theory ranging from classical force fields 7.9 to ab initio methods 10.15 . However, there are still questions that remain to be addressed. In particular, accurately modelling the dynamic nature of the water layer at ambient conditions presents a major obstacle due to the high computational cost of ab initio based methods ${ }^{16 / 17}$. To circumvent this problem, the majority of such studies do not include the liquid water layer explicitly. Instead it is typically replaced by the wetting layer that water is believed to adapt on $\operatorname{Pt}(111)$ at ultra-high-vacuum (UHV) conditions. This structure is typically modelled as an ordered bilayer-like ${ }^{18}$ structure, where the water molecules form a honeycomb $\sqrt{3} \times \sqrt{3} R 30^{\circ}$ pattern with $2 / 3 \mathrm{ML}$ coverage $10-12$. While this structure is supported by some experimental and theoretical evidence $\frac{19}{21}$, it has been a

\footnotetext{
a) Author to whom correspondence should be adressed.
}

subject of debate over the past decades ${ }^{2}$ and the present understanding is that the wetting layer on $\mathrm{Pt}(111)$ is a more complex mixture of pentagonal, hexagonal and heptagonal rings of water molecules $22-24$.

Regardless of whether the bilayer-like wetting layer suggested by 20 provides a reasonable description of the water/Pt(111) interface at UHV, serious doubt may still be raised about whether it provides a realistic framework for modelling it at the relevant operating conditions of electrochemical devices. Consequently, several attempts have been made in recent years at explicitly including the liquid water layer through the use of ab initio molecular dynamics (AIMD) 15, 17] 25, 27. The problem with this is, however, that compared to classical force field approaches, AIMD suffers from large computational requirements, which ultimately limits such studies to small system sizes and short time scales. This can be problematic since it may lead to insufficient thermal sampling of important quantities of interest such as e.g. the structure of the interface ${ }^{26}$. Consequently, methods that can overcome the sampling problems of AIMD while retaining ab initio accuracy would provide an exciting new platform for modelling the water/Pt interface.

In the past decade, machine learning has emerged as a promising framework for circumventing the expensive numerical calculations associated with electronic structure methods 28 . It has been used both for accurate structure prediction across chemical space ${ }^{30 \mid 31}$ as well as for the presently relevant problem of overcoming the sampling limitations of $\mathrm{AIMD}^{32 / 33}$. In particular, it was more recently demonstrated by Natarajan et al ${ }^{33}$ how neural network potentials (NNPs) may be used to describe water on low index $\mathrm{Cu}$ surfaces with ab initio accuracy while retaining the computational cost of classical force fields. A natural question to ask is therefore whether a similar framework may be used to tackle some of the above mentioned challenges in modelling the water/Pt interface. 

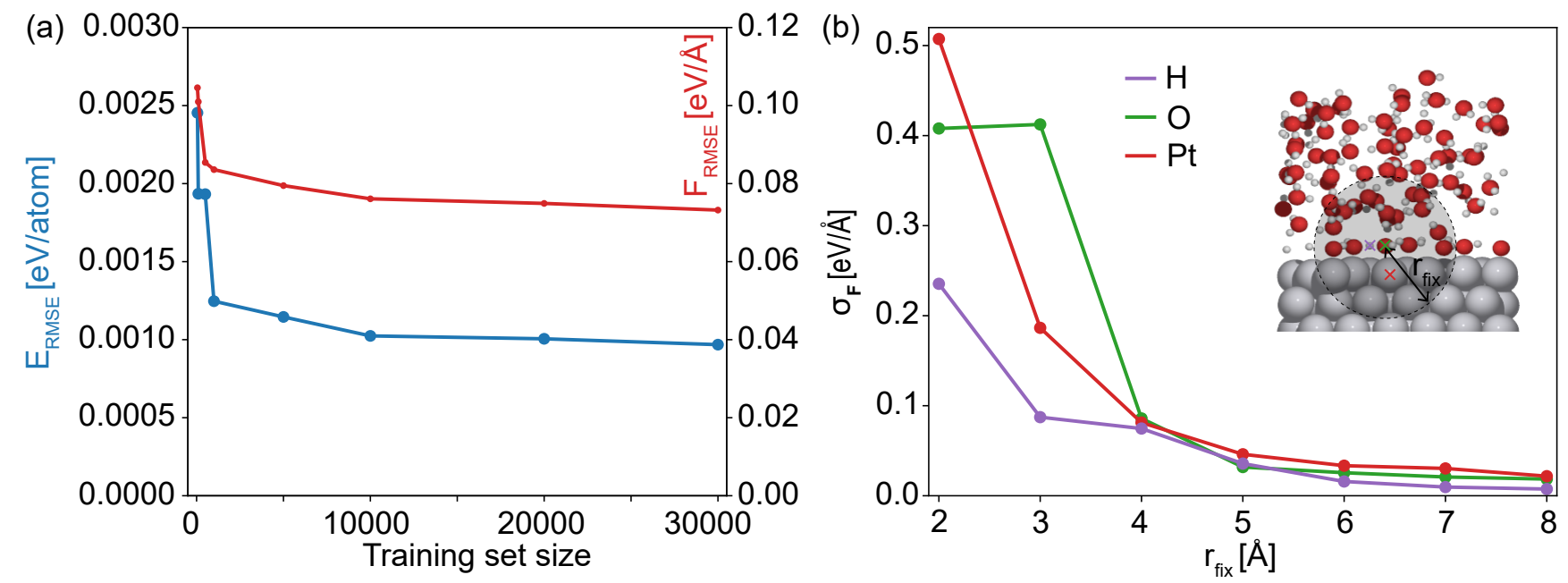

FIG. 1. (a) A learning curve displaying the error in the energies (blue line) and forces (red line) on a fixed test set as the training set is systematically expanded. (b) A measure of the locality of the water/Pt(111) interface based on the procedure of Deringer and Csány 1 .

In this paper we study the water/Pt(111) interface using a machine learning based approach, which rigorously includes the dynamic nature of the liquid water layer, while bridging the problems of high accuracy versus proper sampling. Our main question is: is the water/ $\operatorname{Pt}(111)$ interface ordered at room temperature? We answer this by performing MD simulations with an ensemble of neural network potentials (NNP ensemble), which describe the water/Pt(111) interface with ab initio accuracy while being computationally cheap and thus allowing us to access long time scales. We find that the water/Pt(111) interface is characterized by a double layer composed of a primary adsorption layer with a coverage of $\sim 0.15 \mathrm{ML}$ coupled to a secondary adsorption layer with a coverage of $\sim 0.58 \mathrm{ML}$. Using a lattice based approach we quantify the ordering of the primary adsorption layer and find that there is an effective repulsion between the adsorbed water molecules. This gives rise to a semi-ordered interfacial structure in which the water molecules in the primary adsorption layer are distributed homogeneously across the interface forming frequent hydrogen bonds with the water molecules in the secondary adsorption layer. Finally we analyze the relevant time scales at the interface and find the time scale for transferring water molecules to and from the bulk region to be $\sim 500 \mathrm{ps}$, indicating that our conclusions are outside the scope of conventional AIMD.

\section{METHODS}

\section{A. NNP ensemble}

Our NNP ensemble is a collection of NNPs obtained using the formalism proposed by Behler and Parinelld ${ }^{29}$. In this framework the potential energy surface (PES) of the system is written as a sum of atomic energies

$$
E=\sum_{i=1}^{N} E_{i}\left(\mathbf{G}_{i}\right)
$$

where $\mathbf{G}_{i}$ denotes a vector of 2- and 3-body symmetry functions, which describe the local environment of each atom up to a cutoff radius, $R_{C}{ }^{34}$.

The NNP ensemble consists of three NNPs all fitted using the RuNNer code ${ }^{35 / 36}$, where we employed the same set of symmetry functions as used in a previous NNP study of water on low-index $\mathrm{Cu}$ surfaces ${ }^{33}$. To make the construction of the ensemble as unbiased as possible we trained each NNP based on (i) different random partitionings of our data set into a training (test) set comprising $75 \%$ (25\%) of the available structures, (ii) different random seeds, (iii) different NNP architectures by varying the number of nodes in each hidden layer and the activation function between these. The architecture of each NNP as well as the training and test errors are reported in Table I] The latter are comparable to training and test errors reported for similar studies using NNPs $\$ 33137$.

\begin{tabular}{llll}
\hline & Architecture & $\mathrm{E}_{\text {RMSE }}[\mathrm{eV} /$ atom $]$ & $\mathrm{F}_{\text {RMSE }}[\mathrm{eV} / \AA]$ \\
\hline NNP1 & $30-30(\mathrm{~s}-\mathrm{s}-1)$ & $0.0009(0.0009)$ & $0.076(0.076)$ \\
NNP2 & $30-25(\mathrm{t}-\mathrm{s}-1)$ & $0.0009(0.0009)$ & $0.070(0.070)$ \\
NNP3 & $30-35(\mathrm{t}-\mathrm{s}-1)$ & $0.0011(0.0011)$ & $0.069(0.069)$ \\
\hline
\end{tabular}

TABLE I. Summary of the architecture and training and test errors of the energies and forces for each NNP in our ensemble. For the architectures (first column), the number of nodes in each hidden layer is displayed as $\mathrm{X}-\mathrm{Y}-\mathrm{Z}$ etc. (i.e. 30-30 indicates two hidden layers with 30 nodes in each) and the activation functions used are indicated in parenthesis, where $t, s$ and 1 are abbreviations for tanh, sigmoid and linear, respectively. For the training and test errors (second and third column) the first number is the training error and the second in parenthesis is the test error. 

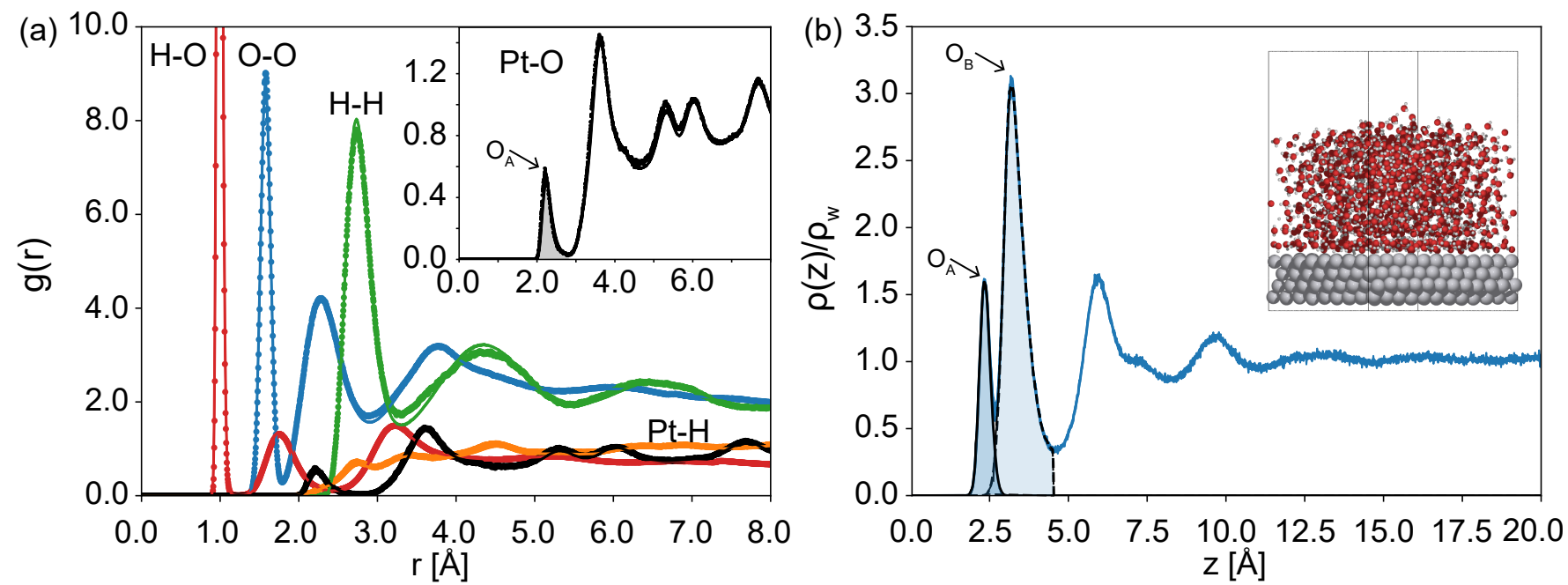

FIG. 2. (a) Different radial distribution functions of selected atomic-type pairs as predicted by AIMD (points) and our NNP (lines). The width of the curves reflects the spread in the predictions of our NNP ensemble. Inset shows a zoom-in highlighting the Pt-O radial distribution function (obtained using the top layer of the $\mathrm{Pt}(111)$ slab only) showing a clear peak due to surface bound oxygen atoms, $\mathrm{O}_{A}$. (b) Planar averaged density profile of water molecules (normalized by the experimental density of bulk water at $75^{\circ} \mathrm{C}$ ) away from the metal slab showing that the interface is characterized by a double peak, which can be separated into water molecules belonging to oxygen atoms of types $\mathrm{O}_{A}$ (strongly bound), $\mathrm{O}_{B}$ (weakly bound).

\section{B. Training database}

All NNP fits were trained on a database of 48041 structures consisting of a $3 \times 4$ orthogonal $\mathrm{Pt}(111)$ slab with a water layer of 32 water molecules. The database was constructed in an iterative manner starting from small AIMD-generated data sets of roughly 1000 structures, which were then gradually expanded by performing MD simulations up to 10ns with preliminary NNP fits and sampling uncorrelated structures from these simulations. This process was done to ensure that the final database of structures was sufficiently dense across the variety of configurations encountered over long time scales. The structures were set up using the Atomistic Simulation Environment (ASE) $\sqrt{38}$, and their energies and forces were determined using the PBE functiona 39 combined with the D3 van der Waals correction $\stackrel{40}{ }$ as implemented in the Vienna ab initio Simulation Package (VASP) ${ }^{41}$. We employed a plane wave basis set with an energy cutoff of $350 \mathrm{eV}$ and $2 \times 2 \times 1 \mathrm{k}$ points.

\section{Model and data set limitations}

Neural network potentials based on the Behler-Parinello formalism have been applied successfully in modelling the properties of a range of systems 42 . Despite this, they are ultimately data-driven and rely on a local expansion of the PES, which inevitably leads to small discrepancies between any NNP-fitted PES and that of the underlying first-principles reference. This observation explains our choice of constructing an ensemble of NNPs, and throughout the remainder of this paper we will use the width of the ensemble predictions as a lower bound for the accuracy one can expect to achieve with our methodology.

To assess the significance of long range interactions and data set limitations further, we have calculated the learning curve for our data set and estimated the long range character of our system. The learning curve was determined by setting aside a fixed test set of roughly 18041 structures chosen randomly from our dataset. The training set was then incrementally expanded using using the remaining structures in the database, while monitoring the error on the forces and energies. As shown in Fig. 1 (a) the error in the forces and energies on the fixed test set drops rapidly as a function of training set size up to around only $\sim 100$ structures after which the improvement is much more modest. At first sight, this would appear to suggest that the construction of a large database of structures is redundant, and that we could have done equally well with a much smaller subset of training structures. We have, however, found it necessary to have a large training set such as the one employed here. Otherwise the MD simulations would not be stable for extended time scales, probably due to the NNP potential giving unreasonable results when it occasionally encounters configurations that are not sufficiently close to numerous points in the training set.

To assess the role of long range interactions, we have estimated the locality of our system using the procedure suggested by Deringer and Csány 1 . In this procedure, a central atom is chosen and every atom within a distance of $r_{\text {fix }}$ are then fixed while atoms outside are distorted randomly. By then monitoring the standard deviation of the force on the central atom, an estimate of the locality of the system is obtained. In our case, we focused on the $\mathrm{O}, \mathrm{H}$ and $\mathrm{Pt}$ atoms shown in Fig. 1 (b) and performed calculations in a $2 \times 2 \times 1$ unit cell compared to the computational cell of ${ }^{16}(1 \times 1 \times 1$ k-points were used for consistency) to be able to investigate values of 
$r_{\text {fix }}$ up to $8 \AA$. Evidently, the standard deviation of the force decays quickly as a function of $r_{\text {fix }}$, but does not go to zero even for values as large as $r_{\mathrm{fix}}=8 \AA$, indicating that our system exhibits some long range character. By closer inspection it is found that the curves saturate at values around $\sim 0.03 \mathrm{eV} / \AA$, and as this value is comparable in magnitude to the saturation value of the force error in Fig. 11(a), we conclude that long range interactions are what limit the quality of our model.

\section{MD simulations}

MD simulations with our NNPs were performed with the Lammps code 45 using the interface provided by the $\mathrm{n} 2 \mathrm{p} 2$ package $46 / 47$. We performed constant temperature MD at $350 \mathrm{~K}$ within the NVT ensemble using a Nosé-Hoover thermostat ${ }^{48 \mid 49}$ with a characteristic damping time of $2 \mathrm{ps}$. We used a slightly elevated temperature for our simulations, as the PBE functional is known to overestimate the melting point of water 50 . An elevated temperature has also previously been employed in similar studies of water/metal interfaces to mimic nuclear quantum effects on the oxygen distribution in water 5152 . Our choice of thermostat coupling, which is significantly longer than the $\sim 40$ fs damping time that was employed in a previous AIMD study of water on $\operatorname{Pt}(111)^{16}$, was chosen to ensure adiabatic decoupling between the thermostat and the intrinsic dynamical properties of the water/Pt(111) interface. To estimate the time scales of the latter, we have analyzed the decay of the velocity autocorrelation function for the water molecules in our simulations. The results of this analysis, which is described in Section A of the SI, shows that the decay happens on a time scale of $\sim 0.5 \mathrm{ps}$, which is significantly faster than the damping time of the thermostat. To be certain that the results reported below are independent on the choice of thermostat and its coupling time, we furthermore tested different choices of the characteristic damping time of our Nosé-Hoover thermostat as well as other types of thermostats with a 2 ps damping time. The result of this analysis, which is described in Section B of the SI, shows that all cases produced very similar results for the neighbor averages and the dynamical time scales reported below. Integration of the classical equations of motion were performed using Verlet integration ${ }^{53}$ with a time step of $0.5 \mathrm{fs}$, and for all simulations we discarded the initial 100ps to ensure proper equilibration. To prevent the rare desorption of water molecules from the water layer onto the opposite side of our Pt slab an elastic wall in the upper part of the vacuum layer was used.

\section{RESULTS AND DISCUSSION}

To validate the quality of our NNP ensemble we analyzed its ability to reproduce the same structural features of the water/Pt(111) interface as predicted by the AIMD studies of Kristoffersen et al. 16 . The results of this analysis are shown in Fig. 2 (a), where we display selected radial distribution functions obtained by performing MD simulations with our NNP ensemble for the same system considered by Ref. 16 consisting of a $8.3 \AA \times 9.6 \AA \times 36 \AA$ unit cell with a 4-layered orthogonal Pt slab and a water layer of 32 molecules. Evidently, there is good agreement between the radial distribution functions obtained from our NNP ensemble (solid lines) and AIMD (points). Furthermore, as shown in the top right inset, the Pt-O RDF exhibits a well-defined peak at $\sim 2.5 \AA$, which suggests a meaningful definition of oxygen atoms bound to the $\mathrm{Pt}(111)$ surface, $\mathrm{O}_{A}$.

Having validated the quality of our NNP ensemble, we now turn to our main question of characterizing the order at the interface. As the first step towards this, we display in Fig. 2 (b), the planar averaged density profile of water molecules as a function of distance from the Pt surface. This was obtained by performing $3 \mathrm{~ns}$ MD simulations with our NNP ensemble in a $33.2 \AA \times 38.6 \AA \times 50 \AA$ unit cell with a water layer of 1024 molecules as shown in the inset. The density is characterized by a double-peaked structure $2-4.5 \AA$ away from the Pt slab followed by a density depletion region after which it decays towards the bulk density of water in an oscillatory fashion. This double-peaked structure has also been observed in AIMD simulations ${ }^{16}$ and in classical force field studies 9 , though in the latter case the relative sizes of the two peaks is reversed and their total surface coverage is predicted to be close to $1 \mathrm{ML}$, which is different from what we find (see below). As shown in Fig. 2(b) the double-peaked density profile close to the Pt slab naturally suggests a definition of the interfacial water layer as being composed of a primary adsorption layer of the $O_{A}$ atoms defined in the inset of 2 (a), which is coupled to a secondary adsorption layer of water molecules with oxygen atoms, $O_{B}$, less strongly bound to the Pt slab. Based on our simulations we have calculated the coverages corresponding to the primary and secondary adsorption layers to be $0.15 \mathrm{ML}$ and $0.58 \mathrm{ML}$, respectively. These values, which compare well with the AIMD studies of Kristoffersen et al. 16 , show that the interfacial structure is different from a bilayer, which would either give rise to two identical peaks with 1/3ML coverage each (traditional bilayer model by Doering et al ${ }^{54}$ ) or a single peak with 2/3ML coverage (co-planar bilayer structure suggested by Ogasawara et al. ${ }^{[20}$ ). We have verified that this conclusion is not simply an artefact of a too high simulation temperature by also performing MD simulations at $300 \mathrm{~K}$. Here we found essentially the same coverages for the two peaks in Fig. 2 (b).

In an attempt to characterize the ordering of the primary adsorption layer in Fig. 2 (b), and motivated by the strong preference of the $O_{A}$ atoms to adsorb atop Pt sites ${ }^{2}$, we have investigated the average number of different types of neighbor pairs between the $O_{A}$ atoms as defined in Fig. 3 (a). The results of our calculations are shown in Table III, where we display the average numbers as predicted by our NNP-based MD simulations and those obtained when the water molecules are distributed randomly on the Pt sites. Evidently the three nearest neighbor averages, $\langle 1 N\rangle,\langle 2 N\rangle,\langle 3 N\rangle$ are suppressed relative to the non-interacting case, while the neighbor pairs $\langle 4 N\rangle$, $\langle 5 N\rangle,\langle 6 N\rangle$ are correspondingly enhanced thus indicating an effective repulsion between the adsorbed water molecules. As shown in Fig. 3 (b), this repulsion manifests itself in a dynamically-changing interface in which the $O_{A}$ atoms (blue) 
(a)

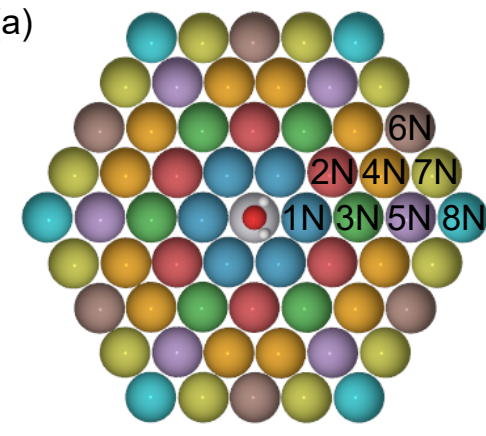

(b)

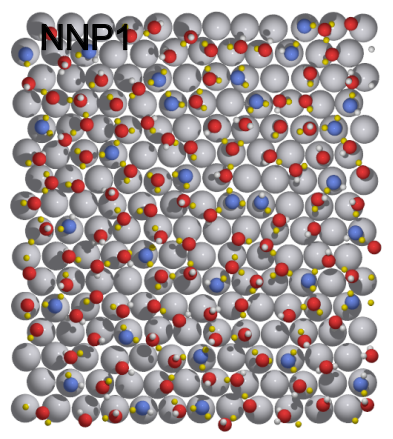

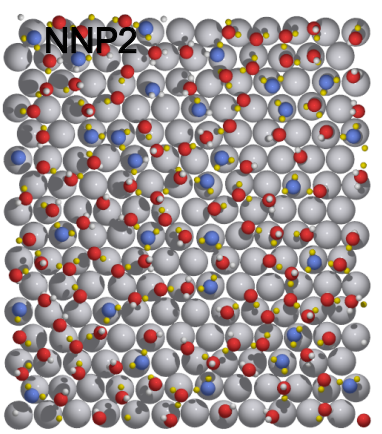

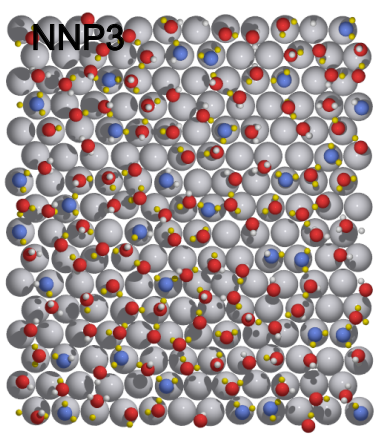

FIG. 3. (a) Illustration of the different types of neighbor pairs in Table II (b) Three snapshots of the interfacial layer chosen randomly from each 1ns MD simulation performed with the three NNPs in our ensemble. Water molecules belonging to the primary adsorption layer are colored blue, and hydrogen atoms participating in a hydrogen bond (we employ the same geometry criterion as used by Ref. 33) are colored gold.

\begin{tabular}{lllllllll}
\hline & $\langle 1 \mathrm{~N}\rangle$ & $\langle 2 \mathrm{~N}\rangle$ & $\langle 3 \mathrm{~N}\rangle$ & $\langle 4 \mathrm{~N}\rangle$ & $\langle 5 \mathrm{~N}\rangle$ & $\langle 6 \mathrm{~N}\rangle$ & $\langle 7 \mathrm{~N}\rangle$ & $\langle 8 \mathrm{~N}\rangle$ \\
\hline NNP1 & $0.40(0.91)$ & $0.54(0.91)$ & $0.52(0.91)$ & $2.38(1.82)$ & $1.32(0.91)$ & $1.03(0.91)$ & $1.90(1.82)$ & $0.82(0.91)$ \\
NNP2 & $0.41(0.92)$ & $0.59(0.92)$ & $0.52(0.92)$ & $2.39(1.85)$ & $1.33(0.92)$ & $1.04(0.92)$ & $1.93(1.85)$ & $0.87(0.92)$ \\
NNP3 & $0.37(0.92)$ & $0.61(0.92)$ & $0.55(0.92)$ & $2.36(1.84)$ & $1.32(0.92)$ & $1.00(0.92)$ & $1.94(1.84)$ & $0.89(0.92)$ \\
\hline
\end{tabular}

TABLE II. Average number of different types of neighbor pairs calculated from MD simulations with each NNP in our ensemble (first number) and when the water molecules are distributed randomly on the Pt sites (second number in parenthesis). The values have been normalized by the number of $O_{A}$ atoms for each structure. The small deviation of the random case for each NNP is due to their slightly different predictions of the $O_{A}$ adsorption layer coverage.

are distributed homogeneously across the interface forming frequent hydrogen bonds (gold-colored $\mathrm{H}$ atoms) with the $O_{B}$ atoms (red) in the secondary adsorption layer. By closer inspection it may also be verified that the interface exhibits characteristic hexagonal bonding patterns similar to those found in conventional bilayers $\frac{20 \mid 54}{2}$ or the experimentally observed lowtemperature $\sqrt{37}$ and $\sqrt{39}$ structures22 24 . We have verified that the results in reported in Table $\Pi$ are robust with respect to starting our simulations from different starting structures of the water overlayer, in particular an ordered bilayer. It should however be noted that while our simulations are thus consistent with a dynamically-changing, semi-ordered interfacial structure, we can of course not rule out that there exists a stable ordered phase at room temperature, which is not observed in our simulations due to nucleation barriers. Another natural question to ask is whether the effective repulsion observed in Table II can be explained from simple geometrical arguments based on the optimization of the number of hydrogen bonds in the primary and secondary adsorption layer. This is not a trivial task. As an example, the number of nearest and nextnearest neighbors in a co-planar bilaye ${ }^{20}$ (which represents a highly optimized structure in terms of number of hydrogen bonds) are suppressed and enhanced, respectively, which is different from our results, where all neighbor pairs in the two inner hexagonal rings of Fig. 3 (a) are suppressed. A plausible physical origin of the effective repulsion is therefore that the atoms in the secondary adsorption layer form energetically favourable shell-like structures around the $O_{A}$ atoms, which extend across the three nearest neighbor sites of Fig. 3 (a).

Would the conclusions above have been obtainable with con- ventional AIMD? Aside from the obvious fact that our NNPs allow us to perform simulations in large unit cells outside the scope of first principles calculations, we have also found the water/Pt(111) interface to exhibit fluctuations on prohibitively long time scales, which must be properly sampled to achieve accurate statistics on the neighbor averages in Table II As a practical demonstration of the problems that this causes for AIMD simulations, we have calculated the average number of nearest $(1 \mathrm{~N})$ and next-nearest neighbors $(2 \mathrm{~N})$ for the $O_{A}$ atoms in the simulation cell employed in the AIMD study of Kristoffersen et al. 16 . The results are displayed in Table III. where we have included the three 50ps AIMD runs conducted by Ref. 16 as well as two $20 \mathrm{~ns}$ runs with each NNP in our ensemble. Evidently the average number of nearest and nextnearest neighbors differ greatly for each AIMD run, while for each NNP they agree within 0.03 neighbors per adsorbed water molecule. We also note that while there is some spread in the predictions across the NNP ensemble, the deviations are small compared to the statistical spread of the AIMD averages, thus demonstrating the general usefulness of NNP ensembles for problems such as the one considered here.

To estimate the typical time scales in our system, we have analyzed the probability for being in the primary adsorption layer, $P_{A}(t)$, the secondary adsorption layer, $P_{B}(t)$, and the bulk water layer, $P_{C}(t)$, based on a three-state model of the 

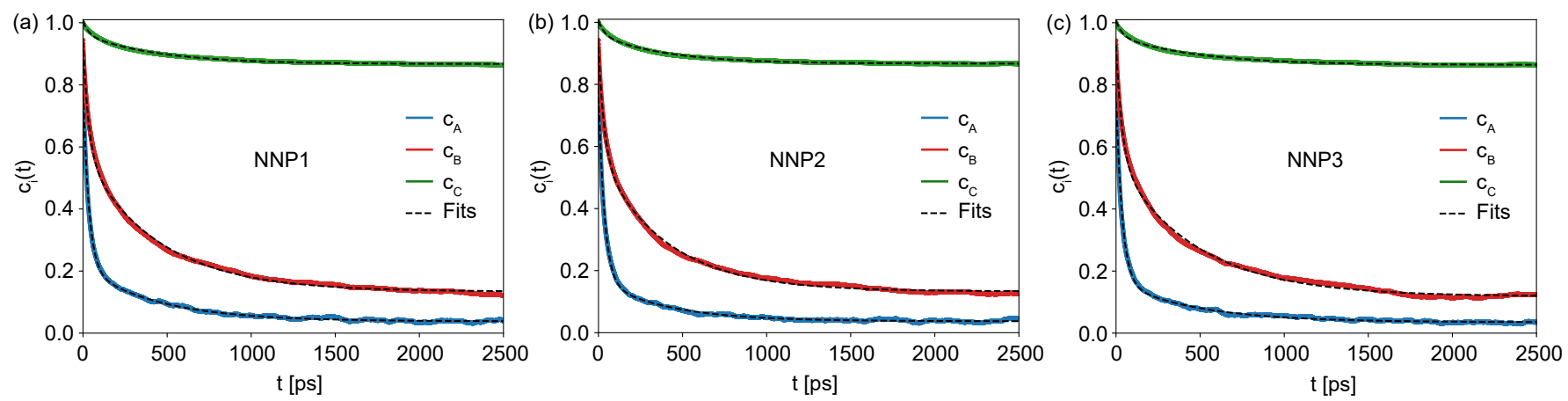

FIG. 4. Values of the residence correlation function in 57 for the A, B and C regions calculated from the MD simulations for each NNP in our ensemble. Fits based on the form (3) are indicated by dashed lines. Extracted fit values are as follows: (a) $k_{+}=0.002 \mathrm{ps}^{-1}, k_{-}=$ $0.030 \mathrm{ps}^{-1}, \mathbf{a}_{0}=(0.04,0.13,0.87)$. (b) $k_{+}=0.003 \mathrm{ps}^{-1}, k_{-}=0.029 \mathrm{ps}^{-1}, \mathbf{a}_{0}=(0.04,0.13,0.87)$. (c) $k_{+}=0.002 \mathrm{ps}^{-1}, k_{-}=0.028 \mathrm{ps}^{-1}, \mathbf{a}_{0}=$ $(0.03,0.12,0.86)$.

\begin{tabular}{lll}
\hline & $\langle 1 \mathrm{~N}\rangle$ & $\langle 2 \mathrm{~N}\rangle$ \\
\hline AIMD run 1 (50ps) & 0.44 & 1.12 \\
AIMD run 2 (50ps) & 0.76 & 0.51 \\
AIMD run 3 (50ps) & 0.28 & 1.49 \\
NNP1 run 1 (20ns) & 0.35 & 0.86 \\
NNP1 run 2 (20ns) & 0.35 & 0.89 \\
NNP2 run 1 (20ns) & 0.33 & 1.02 \\
NNP2 run 2 (20ns) & 0.34 & 1.02 \\
NNP3 run 1 (20ns) & 0.34 & 0.94 \\
NNP3 run 2 (20ns) & 0.34 & 0.97 \\
\hline
\end{tabular}

TABLE III. Average number of nearest and second nearest number of neighbors (normalized per adsorbed water molecule) obtained from three 50ps AIMD runs and two 20ns MD runs with each NNP in our ensemble.

form

$$
\begin{aligned}
& \boldsymbol{P}_{=}^{\prime}=\underset{P}{\boldsymbol{P}} \quad, \quad \boldsymbol{P}=\left(\begin{array}{l}
P_{A}(t) \\
P_{B}(t) \\
P_{C}(t)
\end{array}\right), \\
& \underset{=}{K}=\left(\begin{array}{ccc}
-k_{A B} & k_{B A} & 0 \\
k_{A B} & -\left(k_{B A}+k_{B C}\right) & k_{C B} \\
0 & k_{B C} & -k_{C B}
\end{array}\right),
\end{aligned}
$$

where $k_{X Y}$ is the rate for going from region $\mathrm{X}$ to region $\mathrm{Y}$. The solution to this homogeneous set of differential equations has the form

$$
\boldsymbol{P}(t)=c_{1} \cdot \mathbf{a}_{+} e^{k_{+} t}+c_{2} \cdot \mathbf{a}_{-} e^{k_{-} t}+\mathbf{a}_{0},
$$

where $k_{ \pm}, \mathbf{a}_{ \pm}$denote the non-zero eigenvalues and eigenvectors of $K$ and $c_{1}, c_{2}$ are constants. Finally, the last term is a constant vector, which reflects the steady state probability distribution of water molecules in the three regions:

$$
\mathbf{a}_{0}=1 / N \cdot\left(\begin{array}{c}
\left\langle N_{A}\right\rangle \\
\left\langle N_{B}\right\rangle \\
\left\langle N_{C}\right\rangle
\end{array}\right),
$$

where $\left\langle N_{A}\right\rangle,\left\langle N_{B}\right\rangle,\left\langle N_{C}\right\rangle, N$ denote the average number of water molecules in the $\mathrm{A}, \mathrm{B}$ and $\mathrm{C}$ region, respectively, and $N$ is the total number of water molecules in our simulations.

The non-zero eigenvalues of (3) are given by

$$
\begin{aligned}
& k_{ \pm}=\frac{-k_{1} \pm \sqrt{k_{1}^{2}-4 k_{2}}}{2} \\
& k_{1}=k_{A B}+k_{B A}+k_{B C}+k_{C B} \\
& k_{2}=k_{A B} k_{C B}+k_{B A} k_{C B}+k_{B C} k_{C B}+k_{A B} k_{B C}
\end{aligned}
$$

In general we have found the transfer of water molecules between the $\mathrm{A}$ and $\mathrm{B}$ region to be much faster than transfer to the bulk region such that $k_{A B}, k_{B A}>>k_{B C}, k_{C B}$. In this case one finds to first order $k_{-} \sim-\left(k_{A B}+k_{B A}\right)$ and $k_{+} \approx$ $-\left(k_{C B}+k_{B C} \frac{k_{A B}}{k_{A B}+k_{B A}}\right)$, which shows that $\boldsymbol{P}(t)$ evolves on a fast time scale due to transfer of water molecules between the primary and secondary adsorption layer and a slow time scale due to transfer of water molecules to and from the bulk region.

To utilize the equations above, we have analyzed the residence of oxygen atoms in regions $\mathrm{A}, \mathrm{B}$ and $\mathrm{C}$ by employing correlation functions of the form:

$$
c_{i}(t)=\frac{\left\langle h_{i}(0) h_{i}(t)\right\rangle}{\left\langle h_{i}(0)\right\rangle} .
$$

Here the operator $h_{i}(t)$ is equal to 1 if an $\mathrm{O}$ atom resides in a particular region, indicated by $i$, at time $t$. The notation $\langle\cdot$.$\rangle is used to indicate an average over both simulation$ time and $\mathrm{O}$ atoms in the system. We note that a slightly more strict criterion is often employed in literature ${ }^{33}$, where the atom must have resided in the particular region of interest without interruption until $t$, but we have found that this significantly underestimates the relevant time scales for our system, as $\mathrm{O}$ atoms often make short lived vibrations away from their respective regions. By calculating values of (5) for region A, B and C based on the MD simulations with each of our NNPs and fitting these to the double exponential form in (3) (the same value of $k_{+}$and $k_{-}$ for each region was enforced in the fitting process) we obtain the fits displayed in Fig. 4 Evidently the agreement 
with our three-state model is good, and the asymptotic values found for $\mathbf{a}_{0}$ agree well with the MD-calculated averages $\left(\left\langle N_{A}\right\rangle / N,\left\langle N_{B}\right\rangle / N,\left\langle N_{C}\right\rangle / N \sim(0.03,0.11,0.86)\right.$, which shows that we are able to perform ergodic simulations with the longer time scales accessible to our NNPs. We furthermore see, that all fits agree that the decay of the correlation functions is dominated by a fast time scale on the order of $\tau_{-}=1 / k_{-} \sim 30 \mathrm{ps}$ and slow time scale on the order of $\tau_{+}=1 / k_{+} \sim 500 \mathrm{ps}$. As the latter is beyond the AIMD simulations times that have been employed by the authors of $16 \sqrt[25]{26}$ it demonstrates why longer time scales are needed to achieve proper sampling. We also note, that the observation of a long time scale for surface restructuring agrees well with the classical force field study by Limmer et al. 9 , who estimated the time scale for such reorganizations at the water/Pt(111) interface to be $\sim 1 \mathrm{~ns}$. In their case, this slow organization was, however, attributed to the slow reorientation of the water dipoles, which we do not find to be the physical mechanism.

\section{CONCLUSION AND OUTLOOK}

We have investigated the structure of the water/Pt(111) interface at room temperature using neural network based molecular dynamics simulations, and found the interface to be characterized by a double layer composed of a primary adsorption layer with $\sim 0.15 \mathrm{ML}$ coverage and a secondary, weakly bound layer with a coverage of $\sim 0.58 \mathrm{ML}$. The structure of the primary adsorption layer was analyzed by means of a lattice-based approach, where an effective repulsion between the adsorbed water molecules was found. This repulsion gives rise to a semi-ordered interfacial structure, where the water molecules bound strongly to the interface are distributed homogeneously across the interface forming frequent hydrogen bonds with water molecules in the secondary adsorption layer. Furthermore, we estimated the time scale for transferring water molecules between the primary and secondary adsorption layer to be $\sim 30 \mathrm{ps}$ and the time scale for transfer to and from the bulk region to be $\sim 500 \mathrm{ps,} \mathrm{indicat-}$ ing that our conclusions are outside the scope of conventional AIMD.

Altogether, we believe that this work constitutes an important step towards developing realistic models of solid-liquid interfaces, which are key to a broad range of technological areas. In particular, our framework paves the road towards developing realistic models for catalytic reactions at the water/Pt(111) interface such as e.g. the $\mathrm{OH}$ formation and hydrogen evolution reactions 16 , and we intend to pursue this in future works. Finally, it should be noted that our simulations did not include nuclear quantum effects, which have been shown to have important impact on the structure of water layers at water/metal interfaces 5 , and investigating the effects of these is therefore also an obvious future extension of this work.

\section{SUPPLEMENTARY MATERIAL}

See the supplementary material for an analysis of the velocity autocorrelation function for the water molecules in our system as well as the molecular dynamics simulations conducted with different types of thermostats and damping times.

\section{ACKNOWLEDGEMENTS}

The authors thank Henrik H. Kristoffersen and Heine A. Hansen for stimulating discussions. The authors acknowledge support from the Toyota Research Institute, the Villum Foundations through the research center V-Sustain (grant \#9455) and the Department of Energy Conversion and Storage, Technical University of Denmark, through the Special Competence Initiative Autonomous Materials Discovery (AiMade)

\section{DATA AVAILABILITY}

The data that supports the finding of this study are openly available in the DTU data repository 57 .

${ }^{1}$ V. L. Deringer and G. Csányi, "Machine learning based interatomic potential for amorphous carbon," Phys. Rev. B 95, 094203 (2017)

${ }^{2}$ J. Carrasco, A. Hodgson, and A. Michaelides, "A molecular perspective of water at metal interfaces," Nature Materials 11, 667-674 (2012)

${ }^{3}$ O. Björneholm, M. H. Hansen, A. Hodgson, L.-M. Liu, D. T. Limmer, A. Michaelides, P. Pedevilla, J. Rossmeisl, H. Shen, G. Tocci, E. Tyrode, M.-M. Walz, J. Werner, and H. Bluhm, "Water at interfaces," Chemical Reviews 116, 7698-7726 (2016)

${ }^{4} \mathrm{M}$. K. Debe, "Electrocatalyst approaches and challenges for automotive fuel cells," Nature 486, 43-51 (2012)

${ }^{5}$ J. K. Nørskov, T. Bligaard, A. Logadottir, J. R. Kitchin, J. G. Chen, S. Pandelov, and U. Stimming, "Trends in the exchange current for hydrogen evolution," Journal of The Electrochemical Society 152, J23 (2005)

${ }^{6}$ J. Durst, C. Simon, A. Siebel, P. J. Rheinlander, T. Schuler, M. Hanzlik, J. Herranz, F. Hasche, and H. A. Gasteiger, "(invited) hydrogen oxidation and evolution reaction (HOR/HER) on pt electrodes in acid vs. alkaline electrolytes: Mechanism, activity and particle size effects," ECS Transactions 64, 1069-1080 (2014)

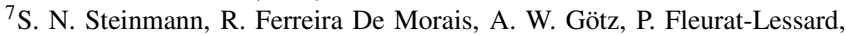
M. Iannuzzi, P. Sautet, and C. Michel, "Force field for water over pt(111): Development, assessment, and comparison," Journal of Chemical Theory and Computation 14, 3238-3251 (2018)

${ }^{\gamma}$ K. Raghavan, K. Foster, K. Motakabbir, and M. Berkowitz, "Structure and dynamics of water at the pt(111) interface: Molecular dynamics study," The Journal of Chemical Physics 94, 2110-2117 (1991)

${ }^{9}$ D. T. Limmer, A. P. Willard, P. Madden, and D. Chandler, "Hydration of metal surfaces can be dynamically heterogeneous and hydrophobic," Proceedings of the National Academy of Sciences 110, 4200-4205 (2013)

${ }^{10}$ J. Rossmeisl, J. K. Nørskov, C. D. Taylor, M. J. Janik, and M. Neurock, "Calculated phase diagrams for the electrochemical oxidation and reduction of water over pt(111)," The Journal of Physical Chemistry B 110, 2183321839 (2006)

${ }^{11}$ E. Skúlason, V. Tripkovic, M. E. Björketun, S. Gudmundsdóttir, G. Karlberg, J. Rossmeisl, T. Bligaard, H. Jónsson, and J. K. Nørskov, "Modeling the electrochemical hydrogen oxidation and evolution reactions on the basis of density functional theory calculations," The Journal of Physical Chemistry C 114, 18182-18197 (2010)

${ }^{12}$ V. Tripković, E. Skúlason, S. Siahrostami, J. K. Nørskov, and J. Rossmeisl, "The oxygen reduction reaction mechanism on $\mathrm{pt}(111)$ from density functional theory calculations," Electrochimica Acta 55, 7975-7981 (2010) 
${ }^{13} \mathrm{~V}$. Tripkovic and T. Vegge, "Potential- and rate-determining step for oxygen reduction on pt(111)," The Journal of Physical Chemistry C 121, 26785$26793(2017)$

${ }^{14} \mathrm{~S}$. Surendralal, M. Todorova, and J. Neugebauer, "Impact of water coadsorption on the electrode potential of h-pt(1 11 1)-liquid water interfaces," Physical Review Letters 126 (2021), 10.1103/physrevlett.126.166802

${ }^{15}$ R. Khatib, A. Kumar, S. Sanvito, M. Sulpizi, and C. S. Cucinotta, "The nanoscale structure of the pt-water double layer under bias revealed," Electrochimica Acta 391, 138875 (2021)

${ }^{16} \mathrm{H}$. H. Kristoffersen, T. Vegge, and H. A. Hansen, "Oh formation and h2 adsorption at the liquid water-pt(111) interface," Chem. Sci. 9, 6912-6921 (2018)

${ }^{17}$ H. H. Kristoffersen, K. Chan, T. Vegge, and H. A. Hansen, "Energy-entropy competition in cation-hydroxyl interactions at the liquid water-pt(111) interface," Chemical Communications 56, 427-430 (2020)

${ }^{18}$ We use the term bilayer-like to indicate that this structure first described by Ogasaware et al $[20$ is similar to the traditional bilayer model proposed by Doering et al ${ }^{54}$ except the fact that all water molecules are co-planar.

${ }^{19}$ T. Schiros, L.-Å. Näslund, K. Andersson, J. Gyllenpalm, G. S. Karlberg, M. Odelius, H. Ogasawara, L. G. M. Pettersson, and A. Nilsson, "Structure and bonding of the water-hydroxyl mixed phase on pt(111)," The Journal of Physical Chemistry C 111, 15003-15012 (2007)

${ }^{20}$ H. Ogasawara, B. Brena, D. Nordlund, M. Nyberg, A. Pelmenschikov, L. G. M. Pettersson, and A. Nilsson, "Structure and bonding of water on pt(111)," Phys. Rev. Lett. 89, 276102 (2002)

${ }^{21} \mathrm{~A}$. Michaelides and P. Hu, "A density functional theory study of hydroxyl and the intermediate in the water formation reaction on pt," The Journal of Chemical Physics 114, 513 (2001)

${ }^{22}$ S. Nie, P. J. Feibelman, N. C. Bartelt, and K. Thürmer, "Pentagons and heptagons in the first water layer on pt(111)," Phys. Rev. Lett. 105, 026102 (2010)

${ }^{23}$ P. J. Feibelman, N. C. Bartelt, S. Nie, and K. Thürmer, "Interpretation of high-resolution images of the best-bound wetting layers on $\mathrm{pt}(111)$, , The Journal of Chemical Physics 133, 154703 (2010)

${ }^{24}$ S. Standop, A. Redinger, M. Morgenstern, T. Michely, and C. Busse, "Molecular structure of theH2owetting layer on pt(111)," Physical Review B 82 (2010), 10.1103/physrevb.82.161412

${ }^{25} \mathrm{~S}$. Sakong, K. Forster-Tonigold, and A. Groß, "The structure of water at a $\mathrm{pt}(111)$ electrode and the potential of zero charge studied from first principles," The Journal of Chemical Physics 144, 194701 (2016)

${ }^{26} \mathrm{~S}$. Schnur and A. Groß, "Properties of metal-water interfaces studied from first principles," New Journal of Physics 11, 125003 (2009)

${ }^{27}$ L. S. Pedroza, A. Poissier, and M.-V. Fernández-Serra, "Local order of liquid water at metallic electrode surfaces," The Journal of Chemical Physics 142, 034706 (2015)

${ }^{28}$ A. P. Bartók, M. C. Payne, R. Kondor, and G. Csányi, "Gaussian approximation potentials: The accuracy of quantum mechanics, without the electrons," Phys. Rev. Lett. 104, 136403 (2010)

${ }^{29} \mathrm{~J}$. Behler and M. Parrinello, "Generalized neural-network representation of high-dimensional potential-energy surfaces," Phys. Rev. Lett. 98, 146401 (2007)

${ }^{30}$ M. Rupp, A. Tkatchenko, K.-R. Müller, and O. A. von Lilienfeld, "Fast and accurate modeling of molecular atomization energies with machine learning," Phys. Rev. Lett. 108, 058301 (2012)

${ }^{31}$ S. De, A. P. Bartók, G. Csányi, and M. Ceriotti, "Comparing molecules and solids across structural and alchemical space," Phys. Chem. Chem. Phys. 18, 13754-13769 (2016)

${ }^{32} \mathrm{Z}$. Li, J. R. Kermode, and A. De Vita, "Molecular dynamics with on-thefly machine learning of quantum-mechanical forces,"'Phys. Rev. Lett. 114, 096405 (2015)

${ }^{33}$ S. K. Natarajan and J. Behler, "Neural network molecular dynamics simulations of solid-liquid interfaces: water at low-index copper surfaces," Phys. Chem. Chem. Phys. 18, 28704-28725 (2016)

${ }^{34} \mathrm{~J}$. Behler, "Atom-centered symmetry functions for constructing highdimensional neural network potentials," The Journal of Chemical Physics 134, 074106 (2011)

${ }^{35}$ J. Behler, "Constructing high-dimensional neural network potentials: A tutorial review," International Journal of Quantum Chemistry 115, 1032-1050
(2015)

${ }^{36} \mathrm{~J}$. Behler, "First principles neural network potentials for reactive simulations of large molecular and condensed systems," Angewandte Chemie International Edition 56, 12828-12840 (2017)

${ }^{3 /}$ B. Cheng, E. A. Engel, J. Behler, C. Dellago, and M. Ceriotti, "Ab initio thermodynamics of liquid and solid water," Proceedings of the National Academy of Sciences 116, 1110-1115 (2019) https://www.pnas.org/content/116/4/1110.full.pdf

${ }^{38}$ A. H. Larsen, J. J. Mortensen, J. Blomqvist, I. E. Castelli, R. Christensen, M. Dułak, J. Friis, M. N. Groves, B. Hammer, C. Hargus, E. D. Hermes, P. C. Jennings, P. B. Jensen, J. Kermode, J. R. Kitchin, E. L. Kolsbjerg, J. Kubal, K. Kaasbjerg, S. Lysgaard, J. B. Maronsson, T. Maxson, T. Olsen, L. Pastewka, A. Peterson, C. Rostgaard, J. Schiøtz, O. Schütt, M. Strange, K. S. Thygesen, T. Vegge, L. Vilhelmsen, M. Walter, Z. Zeng, and K. W. Jacobsen, "The atomic simulation environment-a python library for working with atoms," Journal of Physics: Condensed Matter 29, 273002 (2017)

${ }^{39}$ J. P. Perdew, K. Burke, and M. Ernzerhof, "Generalized gradient approximation made simple," Phys. Rev. Lett. 77, 3865-3868 (1996)

${ }^{40}$ S. Grimme, J. Antony, S. Ehrlich, and H. Krieg, "A consistent and accurate ab initio parametrization of density functional dispersion correction (dft-d) for the 94 elements h-pu," The Journal of Chemical Physics 132, 154104 (2010) https://doi.org/10.1063/1.3382344

${ }^{41}$ G. Kresse and J. Hafner, "Ab initio molecular dynamics for liquid metals," Phys. Rev. B 47, 558-561 (1993)

${ }^{42}$ J. Behler, R. Martoňák, D. Donadio, and M. Parrinello, "Metadynamics simulations of the high-pressure phases of silicon employing a highdimensional neural network potential," Phys. Rev. Lett. 100, 185501 (2008)

${ }^{43}$ G. C. Sosso, G. Miceli, S. Caravati, J. Behler, and M. Bernasconi, "Neural network interatomic potential for the phase change material gete," Phys. Rev. B 85, 174103 (2012)

${ }^{44}$ J. Behler, "First principles neural network potentials for reactive simulations of large molecular and condensed systems," Angewandte Chemie International Edition 56, 12828-12840 (2017)

${ }^{45}$ S. Plimpton, "Fast parallel algorithms for short-range molecular dynamics," Journal of Computational Physics 117, 1 - 19 (1995)

${ }^{46}$ A. Singraber, "CompPhysVienna/n2p2: Neural Network Potential Package," https://doi.org/10.5281/zenodo.1344446(2021).

${ }^{47}$ A. Singraber, J. Behler, and C. Dellago, "Library-based LAMMPS implementation of high-dimensional neural network potentials," Journal of Chemical Theory and Computation 15, 1827-1840 (2019)

${ }^{48} \mathrm{~S}$. Nosé, "A unified formulation of the constant temperature molecular dynamics methods," The Journal of Chemical Physics 81, 511-519 (1984)

${ }^{49}$ W. G. Hoover, "Constant-pressure equations of motion," Physical Review A 34, 2499-2500 (1986)

${ }^{50} \mathrm{~S}$. Du, S. Yoo, and J. Li, "Comparison of the melting temperatures of classical and quantum water potential models," Frontiers in Physics 5 (2017), 10.3389/fphy.2017.00034

${ }^{51}$ M. F. C. Andrade, H.-Y. Ko, L. Zhang, R. Car, and A. Selloni, "Free energy of proton transfer at the water-TiO2 interface from ab initio deep potential molecular dynamics," Chemical Science 11, 2335-2341 (2020)

${ }^{52}$ J. A. Morrone and R. Car, "Nuclear quantum effects in water," Physical Review Letters 101 (2008), 10.1103/physrevlett.101.017801

${ }^{53} \mathrm{~L}$. Verlet, "Computer "experiments" on classical fluids. i. thermodynamical properties of lennard-jones molecules," Phys. Rev. 159, 98-103 (1967)

${ }^{54}$ D. L. Doering and T. E. Madey, "The adsorption of water on clean and oxygen-dosed ru(011)," Surface Science 123, 305-337 (1982)

${ }^{55}$ M. Ceriotti, W. Fang, P. G. Kusalik, R. H. McKenzie, A. Michaelides, M. A. Morales, and T. E. Markland, "Nuclear quantum effects in water and aqueous systems: Experiment, theory, and current challenges," Chemical Reviews 116, 7529-7550 (2016)

${ }^{56}$ http: //aimade.org/

${ }^{57}$ A. E. G. Mikkelsen, J. Schiøtz, T. Vegge, and K. W. Jacobsen, "Dataset and training files for "Is the water/Pt(111) interface ordered at room temperature?","https://doi.org/10.11583/DTU.14791755.v1 (2021). 


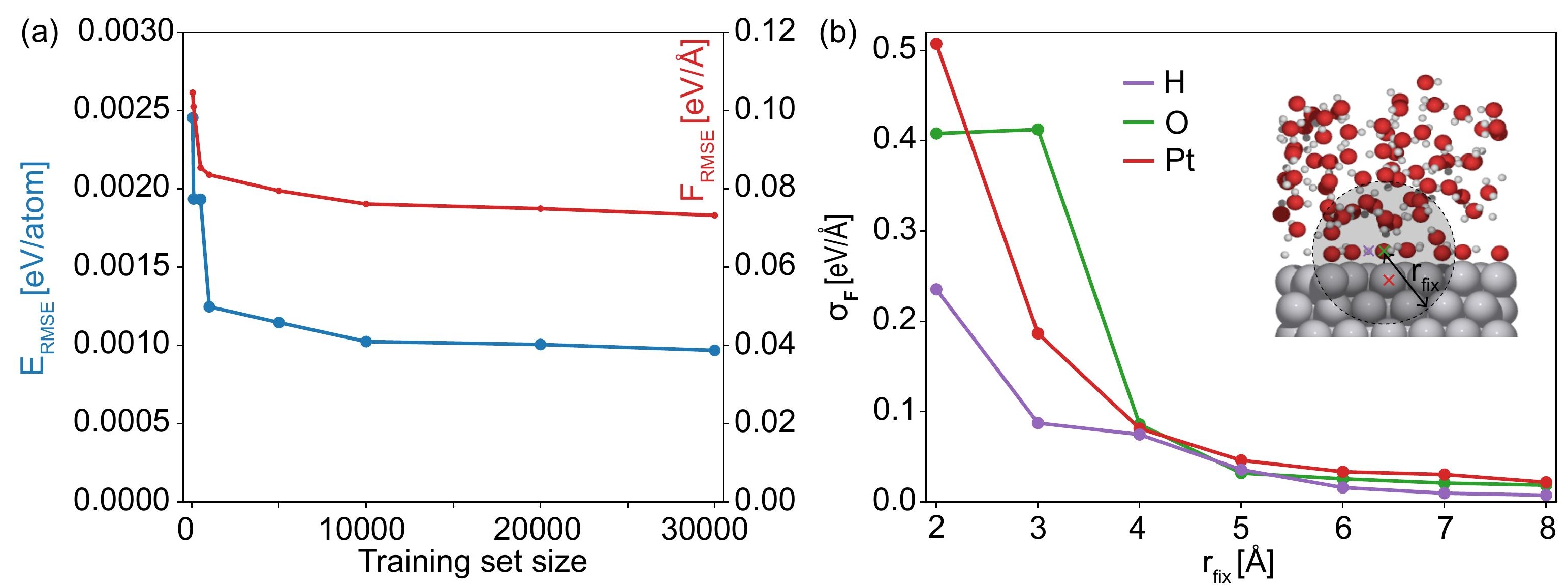


